ETH-TH/1992-18

\title{
Gravity in Non-Commutative Geometry
}

\author{
A. H. Chamseddine ${ }^{1, *}$, G. Felder ${ }^{2}$ and J. Fröhlich ${ }^{3}$ \\ 1 Theoretische Physik, Universität Zürich, CH-8001 Zürich, Switzerland \\ 2 Mathematik, ETH-Zentrum, CH-8092 Zürich, Switzerland \\ 3 Theoretische Physik, ETH-Hönggerberg, CH-8093 Zürich, Switzerland
}

\begin{abstract}
We study general relativity in the framework of non-commutative differential geometry. In particular, we introduce a gravity action for a space-time which is the product of a four dimensional manifold by a two-point space. In the simplest situation, where the Riemannian metric is taken to be the same on the two copies of the manifold, one obtains a model of a scalar field coupled to Einstein gravity. This field is geometrically interpreted as describing the distance between the two points in the internal space.
\end{abstract}

* Supported by the Swiss National Foundation (SNF) 


\section{Introduction}

The poor understanding we have of physics at very short distances might lead to expect that our description of space-time at tiny distances is inadequate. No convincing alternative description is known, but different routes to progress have been proposed. One such proposal is to try to formulate physics on some non-commutative space-time. There appear to be too many possibilities to do this, and it is difficult to see what the right choice is. So the strategy is to consider slight variations of commutative geometry, and to see whether reasonable models can be constructed. This is the approach followed by Connes [1], and Connes and Lott [2,3]. They consider a model of commutative geometry (a Kaluza-Klein theory with an internal space consisting of two points), but use non-commutative geometry to define metric properties. The result is an economical way of deriving the standard model in which, roughly speaking, the Higgs field appears as the component of the gauge field in the internal direction.

In this paper, we show how gravity, in its simplest form, can be introduced in this context. We first propose a generalization of the basic notions of Riemannian geometry. This construction is based on the definition of the Riemannian metric as an inner product on cotangent space. Our definition differs from the one advocated by Connes, who proposes to replace the notion of Riemannian metric by the notion of $K$-cycle. We show however that, for the class of (commutative) Kaluza-Klein models we consider, the two approaches can be related, and each $K$-cycle gives rise to a Riemannian metric in our sense. After this, we propose a generalized Einstein-Hilbert action and see how it looks like in the case of a Kaluza-Klein model with a two-point internal space.

The construction illustrates an interesting feature of non-commutative geometry for commutative spaces: the fact that the metric structure is more general allows one to consider a class of metric spaces more general than Riemannian manifolds, in which however differential geometric notions, such as connections and curvature, still make sense.

The physical picture emerging from this is of a gravitational field described by a Riemannian metric on a four-dimensional space time plus a scalar field which encodes the distance between the two points in the internal space. This field is massless and couples in a minimal way to gravity. Its vacuum expectation value turns out to determine the scale of weak interactions in the formalism of [3]. 


\section{Riemannian geometry}

In this section we develop some concepts of Riemannian geometry in the more general context of non-commutative spaces. Let $\Omega$ be a $\mathbb{Z}$-graded differential algebra over $\mathbb{R}$ or $\mathbb{C}$. This means that $\Omega^{\cdot}=\oplus_{0}^{\infty} \Omega^{n}$ is a graded complex of vector spaces with differential $d: \Omega^{n} \rightarrow \Omega^{n+1}$ and that there is an associative product $m: \Omega^{n} \otimes \Omega^{m} \rightarrow \Omega^{n+m}$. In particular, $A=\Omega^{0}$ is an algebra, and $\Omega^{n}$ is a two sided $A$ module. We will always assume that $\Omega$ has a unit $1 \in A$. The algebra $A$ is to be thought of as a generalization of the algebra of functions on a manifold, and $\Omega$ as a generalization of the space of differential forms. The most important example for us is Connes' algebra of universal forms $\Omega^{\prime}(A)$ over an algebra $A$. It is generated by symbols $f$, of degree zero, and $d f$, of degree one, $f \in A$, with relations $d(f g)=d f g+f d g, f, g \in A$, and $d 1=0$. The notation is consistent since $\Omega^{0}(A)=A$.

To do Riemannian geometry we need a notion of Levi-Civita connection.

In general, a connection on a left $A$ module $E$ is, by definition, a linear map $\nabla: E \rightarrow$ $\Omega^{1} \otimes_{A} E$ such that for any $f \in A$ and $s \in E$,

$$
\nabla(f s)=d f \otimes s+f \nabla s .
$$

For any left $A$ module $E$, define $\Omega E$ to be the graded left $\Omega$ module, $\Omega E=\Omega^{\cdot} \otimes_{A} E$, of " $E$-valued differential forms". A connection $\nabla$ on $E$ extends uniquely to a linear map of degree one $\nabla: \Omega E \rightarrow \Omega \cdot E$ with the property that, for any homogeneous $\alpha \in \Omega, \phi \in \Omega E$,

$$
\nabla(\alpha \phi)=d \alpha \phi+(-1)^{\operatorname{deg}(\alpha)} \alpha \nabla \phi
$$

The curvature of $\nabla$ is then $R(\nabla)=-\nabla^{2}: E \rightarrow \Omega^{2} \otimes_{A} E$, and obeys $-\nabla^{2}(f s)=f\left(-\nabla^{2}\right) s$ for any $f \in A$ and $s \in E$.

Suppose now that $\Omega$ is involutive, i.e. there is an antilinear antiautomorphism $\alpha \mapsto \alpha^{*}$ with $\alpha^{* *}=\alpha$, for all $\alpha \in \Omega$. Assume that $\operatorname{deg}\left(\alpha^{*}\right)=\operatorname{deg}(\alpha)$ and

$(d \alpha)^{*}=(-1)^{\operatorname{deg}(\alpha)+1} d\left(\alpha^{*}\right)$ for homogeneous $\alpha$. If $A$ is any involutive algebra, then the algebra $\Omega^{\cdot}(A)$ of universal differential forms is involutive, with the above properties, if we set $(d f)^{*}=-d\left(f^{*}\right)$ for $f \in A$. In general, elements of $A$ of the form $g=f^{*} f$, are called non-negative $(g \geq 0)$. The module $E$ is called hermitian if it has a hermitian inner product $():, E \times E \rightarrow A$, which is by definition a sesquilinear form such that

(i) $(f s, g t)=f(s, t) g^{*}, \quad f, g \in A, \quad s, t \in E$

(ii) $(s, s) \geq 0$.

(iii) The map $s \mapsto(s, \cdot)$ from $E$ to the left $A$ module $E^{*}=\{l: E \rightarrow A, \quad l(f s+g t)=$ $\left.l(s) f^{*}+l(t) g^{*}\right\}$ is an isomorphism. 
Any hermitian inner product on $E$ extends uniquely to a sesquilinear map $\Omega \cdot E \times \Omega \rightarrow \Omega$. such that $(\alpha \phi, \beta \psi)=\alpha(\phi, \psi) \beta^{*}$ for all $\alpha, \beta \in \Omega, \phi, \psi \in \Omega E$. A connection $\nabla$ on a hermitian $A$-module $E$ is unitary if, for all $s, t \in E, d(s, t)=(\nabla s, t)-(s, \nabla t)$ (the minus sign appears here because we have set $\left.(d f)^{*}=-d f^{*}\right)$. One has then for homogeneous $\phi$, $\psi \in \Omega E$

$$
d(\phi, \psi)=(\nabla \phi, \psi)-(-1)^{\operatorname{deg}(\phi) \operatorname{deg}(\psi)}(\phi, \nabla \psi)
$$

Let us now suppose that $\Omega$ is an algebra over $\mathbb{R}$, and take $E$ to be $\Omega^{1}$. This is the setting of Riemannian geometry.

The torsion of a connection $\nabla: \Omega^{1} \rightarrow \Omega^{1} \otimes \Omega^{1}$ is

$$
T(\nabla)=d-m \circ \nabla
$$

It is an $A$ linear operator from $\Omega^{1}$ to $\Omega^{2}$. The connections of interest in Riemannian geometry are those with vanishing torsion. Among these connections we should like to find ones that can be interpreted as natural generalizations of Levi-Civita connections. This suggests to introduce the notion of a metric in non-commutative geometry. One might think that a metric is specified by an inner product on $\Omega^{1}$. However, in general, it does not appear to make sense to demand this inner product to be hermitian. Actually, it looks more promising to first introduce the notion of a distance on a non-commutative space, as proposed by Connes and Lott [3].

Apparently, in non-commutative geometry the natural notion of distance is provided by $K$-cycles. Recall that a $K$-cycle over an involutive algebra $A$ is a pair $(H, D)$, where $H=H_{+} \oplus H_{-}$is a $\mathbb{Z}_{2}$ graded Hilbert space with a $*$-action of $A$ by even bounded operators, and $D$ is a possibly unbounded, odd self-adjoint operator, called Dirac operator, such that $[D, f]$ is bounded for all $f \in A$ and $\left(D^{2}+1\right)^{-1}$ is compact. Then $\pi\left(f_{0} d f_{1} \cdots d f_{n}\right)=$ $f_{0}\left[D, f_{1}\right] \cdots\left[D, f_{n}\right]$ defines an involutive (i.e. with $\left.\pi\left(\alpha^{*}\right)=\pi(\alpha)^{*}\right)$ representation of the algebra $\Omega(A)$ of universal forms. One shows then that the graded subcomplex $\operatorname{Ker}(\pi)+$ $d \operatorname{Ker}(\pi)$ is a two-sided ideal of $\Omega^{\prime}(A)$, so that the quotient

$$
\Omega_{D}(A)=\Omega^{\cdot}(A) /(\operatorname{Ker}(\pi)+d \operatorname{Ker}(\pi))
$$

is a graded differential algebra ${ }^{1}$.

A Riemannian metric is a hermitian inner product - more generally, a non-degenerate inner product - on $\Omega_{D}^{1} \equiv \Omega_{D}^{1}(A)$ which (in the examples considered below) determines a

1 This algebra was introduced in the Cargèse lecture notes of Connes and Lott [3]. It replaces the algebra of universal forms used in $[1,2]$ and allows for a more transparent treatment of "auxiliary fields". 
notion of distance coinciding with the one obtained from the Dirac operator, as in [3]. A connection $\nabla$ on $\Omega^{1}$ is a Levi-Civita connection if it has vanishing torsion and if $\pi(\nabla)$ is unitary with respect to the metric on $\Omega_{D}^{1}$. In general it is not true, as it is in the classical case, that for an arbitrary Riemannian metric there is precisely one Levi-Civita connection.

It is straightforward to derive Cartan structure equations in this context. Suppose that $\Omega_{D}^{1}$ is a trivial vector bundle, i.e. a free, finitely generated $A$ module, with Riemannian metric. (The following analysis could be generalized to situations where $\Omega_{D}^{1}$ is a nontrivial vector bundle by introducing a suitable family of $\Omega_{D}^{1}$-invariant subspaces of $H$, with the property that the restriction of $\Omega_{D}^{1}$ to every subspace in this family is trivial.) Let $E^{A}, A=1, \ldots, N$, be a basis of sections of $\Omega_{D}^{1}$ which is orthonormal in the metric on $\Omega_{D}^{1}$. We define $\Omega^{A B} \in \Omega_{D}^{1}$ by

$$
\pi(\nabla) E^{A}=-\sum_{B} \Omega^{A B} \otimes E^{B} .
$$

The components of torsion and curvature are defined by

$$
\begin{aligned}
& \pi(T(\nabla)) E^{A}=T^{A} \\
& \pi(R(\nabla)) E^{A}=\sum_{B} R^{A B} \otimes E^{B} .
\end{aligned}
$$

The Cartan structure equations follow by inserting the definitions of $T(\nabla)$ and $R(\nabla)$ :

$$
\begin{aligned}
T^{A} & =\pi\left(d \tilde{E}^{A}\right)+\sum_{B} \Omega^{A B} E^{B}, \\
R^{A B} & =\pi\left(d \tilde{\Omega}^{A B}\right)+\sum_{C} \Omega^{A C} \Omega^{C B},
\end{aligned}
$$

where $\tilde{E}^{A}, \tilde{\Omega}^{A B}$ are representatives of $E^{A}, \Omega^{A B}$, respectively, in $\Omega^{1}$.

We now introduce a class of algebras and of $K$-cycles for which the Riemannian geometry concepts introduced above can be defined.

Let $X$ be a compact even dimensional $C^{\infty}$ spin manifold, with a reference Riemannian metric $g_{0}$ and fixed spin structure, $A$ the algebra of smooth real functions on $X$ and Cliff $\left(T^{*} X\right)$ the Clifford bundle over $X$, whose fiber at $x$ is the (real) Clifford algebra of the cotangent space $\operatorname{Cliff}\left(T_{x}^{*} X\right)$ associated to $g_{0}(x)$. Let $S$ be the spinor bundle. Thus $S$ is a $\mathbb{Z}_{2}$ graded complex vector bundle over $X$, with a representation of the Clifford algebra of the cotangent space on each fiber $S_{x}$, such that $\operatorname{End}_{\mathbb{C}}\left(S_{x}\right) \simeq \operatorname{Cliff}\left(T_{x}^{*} X\right) \otimes \mathbb{C}$. A section of $\operatorname{End}(S) \simeq \operatorname{Cliff}\left(T^{*} X\right) \otimes \mathbb{C}$ is called real if it takes values in the real Clifford algebra. We consider $K$-cycles $(H, D)$ where: 
(i) $D$ is an odd first order elliptic differential operator on the space $C^{\infty}(S)$ of smooth sections of $S$.

(ii) For each $f \in A,[D, f]$ is a real section of $\operatorname{End}(S)$.

(iii) $H=L^{2}\left(S, \rho d^{4} y\right)$ is the space of square integrable sections of $S$, where $\rho(y)$ is a density for which $D$ is self-adjoint.

We will also need the following variant with group action: Let $X, A, \operatorname{Cliff}\left(T^{*} X\right)$ and $S$ be as above, and suppose that $X$ is a finite smooth covering of a manifold $Y$. That is, $p: X \rightarrow Y$ is a principal $G$ bundle with base space $Y=X / G$, and $G$ is a finite group. The reference metric will be chosen to be preserved by the group action, and we assume that the group action lifts to $S$. Denote by $p_{*} S$ the vector bundle over $Y$ whose fiber over $y$ is the direct sum $\oplus_{p(x)=y} S_{x}$. Both $A$ and the group $G$ act on the sections of $p_{*} S$. A linear operator on the space of smooth section of $p_{*} S$ is called equivariant if it commutes with the action of $G$. The vector space $\operatorname{End}_{\mathbb{C}}\left(p_{*} S_{y}\right)$ is the space of matrices indexed by $p^{-1}(y)$ with entries in $\operatorname{Cliff}\left(T_{y}^{*} Y\right) \otimes \mathbb{C}$. A vector in $\operatorname{End}_{\mathbb{C}}\left(p_{*} S_{y}\right)$ is called real if its matrix entries belong to the real Clifford algebra, and a section of $\operatorname{End}\left(p_{*} S\right)$ is called real if it takes real values.

In this setting, we consider $K$-cycles $(H, D)$ where:

(i) $D$ is an odd equivariant first order elliptic differential operator on the space $C^{\infty}\left(p_{*} S\right)$ of smooth sections of $p_{*} S$.

(ii) For each $f \in A,[D, f]$ is multiplication by a real section of $\operatorname{End}\left(p_{*} S\right)$

(iii) $H=L^{2}\left(p_{*} S, \rho d^{4} y\right)$ is the space of square integrable sections of $p_{*} S$, where $\rho(y)$ is a density for which $D$ is self-adjoint.

These data define a Riemannian geometry on the graded differential algebra $\Omega_{D}^{*}(A)$. The Riemannian metric is defined to be

$$
G(\alpha, \beta)=\operatorname{tr}\left(\pi\left(\alpha^{*}\right) \pi(\beta)\right), \quad \alpha, \beta \in \Omega_{D}^{1}(A)
$$

This is independent of the choice of representatives $\alpha, \beta$ since $d \operatorname{Ker}(\pi) \cap \Omega^{1}(A)=0$ and therefore $\Omega_{D}^{1}(A)$ is isomorphic to $\pi\left(\Omega^{1}(A)\right)$. The trace over the Clifford algebra is defined fiberwise. We normalize it in such a way that the trace of the identity is one. 


\section{A gravity action}

Let us apply the formalism introduced in the previous section to an example. As in [1-3], we take $X$ to be two copies of a compact, say four-dimensional, spin manifold $Y$ :

$$
X=Y \times \mathbb{Z}_{2}
$$

and we have the trivial $\mathbb{Z}_{2}$ bundle $p: X \rightarrow Y$. The algebra $A$ is then $C_{\mathbb{R}}^{\infty}(Y) \oplus C_{\mathbb{R}}^{\infty}(Y)$. It is convenient to think of $A$ as a subalgebra of diagonal matrices in the algebra $M_{2}(\mathbb{C}) \otimes$ $C^{\infty}\left(\operatorname{Cliff}\left(T^{*} Y\right)\right)$ of two by two matrices whose entries are smooth sections of the Clifford bundle. The chirality operator $\gamma^{5}$ belongs to the real Clifford algebra and defines a $\mathbb{Z}_{2}$ grading of the spinor bundle $S$. The operator

$$
\Gamma=\left(\begin{array}{cc}
\gamma^{5} & 0 \\
0 & -\gamma^{5}
\end{array}\right)
$$

defines a $\mathbb{Z}_{2}$ grading of $C^{\infty}\left(p_{*} S\right)=C^{\infty}(S) \oplus C^{\infty}(S)$ (the minus sign is a matter of convention).

We work in local coordinates. Let us introduce gamma matrices $\gamma^{a}$ with $\left(\gamma^{a}\right)^{*}=-\gamma^{a}$, $a=1, \ldots, 4$, obeying the relations $\gamma^{a} \gamma^{b}+\gamma^{b} \gamma^{a}=-2 \delta^{a b}$. Then $\gamma^{5}=\gamma^{1} \gamma^{2} \gamma^{3} \gamma^{4}$ is self-adjoint and has square one. We set $\gamma^{a b}=\frac{1}{2}\left(\gamma^{a} \gamma^{b}-\gamma^{b} \gamma^{a}\right)=-\left(\gamma^{b a}\right)^{*}$.

The Dirac operator can then be represented as a two by two matrix $\left(D_{i j}\right), i, j \in$ $\{+,-\}$, whose entries are first order differential operators acting on spinors of $Y$. What are the restrictions on these entries imposed by (i)-(iii)? First of all, $\mathbb{Z}_{2}$ equivariance implies that $D_{+-}=D_{-+}$and $D_{++}=D_{--}$, and the fact that $[D, f]$ is a multiplication operator implies that $D_{+-}$should be a multiplication operator. The most general form of $D$, compatible with self-adjointness, reality and oddness is then

$$
D=\left(\begin{array}{cc}
\gamma^{a} \epsilon_{a}^{\mu} \partial_{\mu}+\cdots & \psi+\gamma^{5} \phi \\
\psi+\gamma^{5} \phi & \gamma^{a} \epsilon_{a}^{\mu} \partial_{\mu}+\cdots
\end{array}\right)
$$

where $\epsilon_{a}^{\mu}, \psi$ and $\phi$ are real functions. Since $D$ is elliptic, $\epsilon_{a}^{\mu} \partial_{\mu}$ is a basis of the tangent space, and we can define a Riemannian metric $g$ on $Y$ by $g\left(\epsilon_{a}, \epsilon_{b}\right)=\delta_{a b}$. The dots in the definition of $D$ indicate zero order contributions which do not contribute to $\pi$.

The representation $\pi$ on one-forms can now be computed. Let $\alpha=\Sigma_{i} a_{i} d b_{i} \in \Omega^{1}(A)$ be a representative of a one-form in $\Omega_{D}^{1}(A)$. Then $\pi(\alpha)$ is parametrized by two classical one-forms $\alpha_{1 \mu}, \alpha_{2 \mu}$, and two functions $\alpha_{5}, \tilde{\alpha}_{5}$, on $Y$ :

$$
\pi(\alpha)=\left(\begin{array}{cc}
\gamma^{\mu} \alpha_{1 \mu} & \bar{\gamma} \alpha_{5} \\
-\bar{\gamma} \tilde{\alpha}_{5} & \gamma^{\mu} \alpha_{2 \mu}
\end{array}\right)
$$


We use the notation $\gamma^{\mu}=\gamma^{a} \varepsilon_{a}^{\mu}, \bar{\gamma}=\psi+\gamma^{5} \phi$. In terms of the variables $a_{i}=a_{i 1} \oplus a_{i 2}$ and $b_{i}=b_{i 1} \oplus b_{i 2}$, we have

$$
\begin{aligned}
\alpha_{1 \mu} & =\sum_{i} a_{i 1} \partial_{\mu} b_{i 1}, \\
\alpha_{2 \mu} & =\sum_{i} a_{i 2} \partial_{\mu} b_{i 2}, \\
\alpha_{5} & =\sum_{i} a_{i 1}\left(b_{i 2}-b_{i 1}\right), \\
\tilde{\alpha}_{5} & =\sum_{i} a_{i 2}\left(b_{i 2}-b_{i 1}\right),
\end{aligned}
$$

The Riemannian metric $G: \Omega_{D}^{1}(A) \otimes \Omega_{D}^{1}(A) \rightarrow A$ can be expressed, using the isomorphism $\Omega_{D}^{1}(A)=\pi\left(\Omega^{1}(A)\right)$, in terms of components:

$$
G(\alpha, \beta)=\left(g^{\mu \nu} \alpha_{1 \mu} \beta_{1 \nu}+g^{55} \tilde{\alpha}_{5} \tilde{\beta}_{5}\right) \oplus\left(g^{\mu \nu} \alpha_{2 \mu} \beta_{2 \nu}+g^{55} \alpha_{5} \beta_{5}\right),
$$

where $g^{\mu \nu}=-\operatorname{tr}\left(\gamma^{\mu} \gamma^{\nu}\right)=\epsilon_{a}^{\mu} \epsilon_{a}^{\nu}$ and $g^{55}=\operatorname{tr} \bar{\gamma}^{2}=\psi^{2}+\phi^{2}$.

To compute torsion and curvature, we must understand two-forms, $\Omega_{D}^{2}(A)$. This space is isomorphic to the quotient of $\pi\left(\Omega^{2}(A)\right)$ by the space of "auxiliary fields" $\pi\left(d \operatorname{Ker}\left(\left.\pi\right|_{\Omega^{1}(A)}\right)\right)$. We proceed to compute the general form of auxiliary fields. If $\alpha=$ $\Sigma_{i} a_{i} d b_{i} \in \operatorname{Ker}(\pi)$, we obtain for $\pi(d \alpha)=\Sigma_{i}\left[D, a_{i}\right]\left[D, b_{i}\right]$,

$$
\pi(d \alpha)=\left(\begin{array}{ll}
-g^{\mu \nu} \partial_{\mu} a_{i 1} \partial_{\nu} b_{i 1} & -2 \psi \gamma^{\mu} a_{i 1} \partial_{\mu} b_{i 2} \\
-2 \psi \gamma^{\mu} a_{i 2} \partial_{\mu} b_{i 1} & -g^{\mu \nu} \partial_{\mu} a_{i 2} \partial_{\nu} b_{i 2}
\end{array}\right)
$$

and it is not difficult to see that, for a suitable choice of $a_{i}, b_{i}$ subject to the constraint $\pi(\alpha)=0$, any expression of the form

$$
\left(\begin{array}{cc}
X_{1} & \psi \gamma^{\mu} Y_{\mu} \\
\psi \gamma^{\mu} \tilde{Y}_{\mu} & X_{2}
\end{array}\right)
$$

can be obtained.

Next, we express $\pi(d \alpha)$ modulo auxiliary fields for any one-form $\alpha$ in terms of its components:

$$
\pi(d \alpha)=\left(\begin{array}{cc}
\gamma^{\mu \nu} \partial_{\mu} \alpha_{1 \nu}+2 \phi \psi \gamma^{5}\left(\alpha_{5}-\tilde{\alpha}_{5}\right) & \phi \gamma^{\mu} \gamma^{5}\left(\partial_{\mu} \alpha_{5}+\alpha_{1 \mu}-\alpha_{2 \mu}\right) \\
-\phi \gamma^{\mu} \gamma^{5}\left(\partial_{\mu} \tilde{\alpha}_{5}+\alpha_{1 \mu}-\alpha_{2 \mu}\right) & \gamma^{\mu \nu} \partial_{\mu} \alpha_{2 \nu}+2 \phi \psi \gamma^{5}\left(\alpha_{5}-\tilde{\alpha}_{5}\right)
\end{array}\right)
$$

This choice of representative in the class of $\pi(d \alpha)$ in $\pi\left(\Omega^{2}(A)\right) / \pi\left(d \operatorname{Ker}\left(\left.\pi\right|_{\Omega^{1}(A)}\right)\right)$ is uniquely determined by the property to be orthogonal to all auxiliary fields, with respect to the inner product on $\Omega^{2}(A)$ defined by the Dixmier trace:

$$
(\alpha, \beta)=\operatorname{Tr}_{\omega}\left(\pi(\alpha)^{*} \pi(\beta)|D|^{-4}\right)
$$


For explicit calculations it is convenient to introduce local orthonormal bases $\left\{E^{A}\right\}$ of $\Omega_{D}^{1}(A)$. We use the following convention for indices: capital letters $A, B$, .. denote indices taking the values 1 to 5 , and lower case letters $a, b, \ldots$ take values from 1 to 4 . Introduce a local orthonormal frame of one-forms $e_{\mu}^{a} d x^{\mu}$ on $Y$. The basis is

$$
\begin{aligned}
& E^{a}=\left(\begin{array}{cc}
\gamma^{a} & 0 \\
0 & \gamma^{a}
\end{array}\right)=\left(\begin{array}{cc}
\gamma^{\mu} e_{\mu}^{a} & 0 \\
0 & \gamma^{\mu} e_{\mu}^{a}
\end{array}\right), \\
& E^{5}=\left(\begin{array}{cc}
0 & \bar{\gamma} \lambda \\
-\bar{\gamma} \lambda & 0
\end{array}\right), \quad \lambda=\left(\phi^{2}+\psi^{2}\right)^{-\frac{1}{2}}
\end{aligned}
$$

Suppose now that the connection $\nabla$ is unitary with respect to the given $K$-cycle. The components of the one-form corresponding to $\pi(\nabla)$ are denoted by

$$
\Omega^{A B}=\left(\begin{array}{cc}
\gamma^{\mu} \omega_{1 \mu}^{A B} & \bar{\gamma} \ell^{A B} \\
-\bar{\gamma} \tilde{\ell}^{A B} & \gamma^{\mu} \omega_{2 \mu}^{A B}
\end{array}\right)
$$

The unitarity condition $\left(\Omega^{A B}\right)^{*}=\Omega^{B A}$ implies the component relations

$$
\begin{gathered}
\omega_{1 \mu}^{A B}=-\omega_{1 \mu}^{B A} \\
\omega_{2 \mu}^{A B}=-\omega_{2 \mu}^{B A} \\
\tilde{\ell}^{A B}=-\ell^{B A}
\end{gathered}
$$

The components of torsion and curvature are readily computed. As above, we give the representative in $\Omega_{D}^{2}(A)$ orthogonal to auxiliary fields. For the torsion we find

$$
\begin{aligned}
T^{a} & =\left(\begin{array}{cc}
\gamma^{\mu \nu}\left(\partial_{\mu} e_{\nu}^{a}+\omega_{1 \mu}^{a b} e_{\nu}^{b}\right)-2 \phi \psi \lambda \gamma^{5} \ell^{a 5} & -\phi \gamma^{\mu} \gamma^{5}\left(\ell^{a b} e_{\mu}^{b}-\lambda \omega_{1 \mu}^{a 5}\right) \\
\phi \gamma^{\mu} \gamma^{5}\left(\tilde{\ell}^{a b} e_{\mu}^{b}-\lambda \omega_{2 \mu}^{a 5}\right) & \gamma^{\mu \nu}\left(\partial_{\mu} e_{\nu}^{a}+\omega_{2 \mu}^{a b} e_{\nu}^{b}\right)-2 \phi \psi \lambda \gamma^{5} \tilde{\ell}^{a 5}
\end{array}\right), \\
T^{5} & =\left(\begin{array}{cc}
\gamma^{\mu \nu} \omega_{1 \mu}^{5 b} e_{\nu}^{b}-2 \phi \psi \lambda \gamma^{5} \ell^{55} & \phi \gamma^{\mu} \gamma^{5}\left(\partial_{\mu} \lambda-\ell^{5 b} e_{\mu}^{b}\right) \\
-\phi \gamma^{\mu} \gamma^{5}\left(\partial_{\mu} \lambda-\tilde{\ell}^{5 b} e_{\mu}^{b}\right) & \gamma^{\mu \nu} \omega_{2 \mu}^{5 b} e_{\nu}^{b}+2 \phi \psi \lambda \gamma^{5} \ell^{55}
\end{array}\right) .
\end{aligned}
$$

The expression for the curvature is

$$
R^{A B}=\left(\begin{array}{cc}
\gamma^{\mu \nu} R_{1 \mu \nu}^{A B}+2 \phi \psi \gamma^{5} P_{1}^{A B} & \phi \gamma^{\mu} \gamma^{5} Q_{\mu}^{A B} \\
-\phi \gamma^{\mu} \gamma^{5} \tilde{Q}_{\mu}^{A B} & \gamma^{\mu \nu} R_{2 \mu \nu}^{A B}+2 \phi \psi \gamma^{5} P_{2}^{A B}
\end{array}\right)
$$

where

$$
\begin{aligned}
& R_{i \mu \nu}=\partial_{\mu} \omega_{i \nu}^{A B}-\partial_{\nu} \omega_{i \mu}^{A B}+\omega_{i \mu}^{A C} \omega_{i \nu}^{C B}-\omega_{i \nu}^{A C} \omega_{i \mu}^{C B}, \quad i=1,2, \\
& Q_{\mu}^{A B}=\partial_{\mu} \ell^{A B}+\omega_{1 \mu}^{A B}-\omega_{2 \mu}^{A B}+\omega_{1 \mu}^{A C} \ell^{C B}-\omega_{2 \mu}^{C B} \ell^{A C}, \\
& \tilde{Q}_{\mu}^{A B}=-\partial_{\mu} \ell^{B A}+\omega_{1 \mu}^{A B}-\omega_{2 \mu}^{A B}+\omega_{1 \mu}^{C B} \ell^{C A}-\omega_{2 \mu}^{A C} \ell^{B C}, \\
& P_{1}^{A B}=\ell^{A B}+\ell^{B A}+\ell^{A C} \ell^{B C} \\
& P_{2}^{A B}=\ell^{A B}+\ell^{B A}+\ell^{C A} \ell^{C B}
\end{aligned}
$$


As a gravity action we propose the following generalized Einstein-Hilbert action, given in terms of the inner product $(\alpha, \beta)=\operatorname{Tr}_{\omega}\left(\pi(\alpha)^{*} \pi(\beta)|D|^{-4}\right)$ on two-forms defined through the identification of $\Omega_{D}^{2}(A)$ with $\pi\left(\Omega^{2}(A)\right) \cap \pi\left(d \operatorname{Ker}\left(\left.\pi\right|_{\Omega^{1}(A)}\right)^{\perp}\right)$ :

$$
I=\left(E^{A} E^{B}, R^{A B}\right)
$$

This action reduces to (and could be alternatively defined as) the integral over $Y$,

$$
I=\int_{Y} \operatorname{tr}\left(\left(E^{A} E^{B}\right)^{*} R^{A B}\right) \sqrt{g} d^{4} y
$$

(Here the trace is over $\operatorname{End}\left(p_{*} S_{y}\right)$ ). Inserting the above expressions for $E^{A}$ and $R^{A B}$ yields the action as a function of the component fields. Set $U_{\mu}^{a}=Q_{\mu}^{a 5}+\tilde{Q}_{\mu}^{a 5}-Q_{\mu}^{5 a}-\tilde{Q}_{\mu}^{5 a}$. The result is

$$
I=\int_{Y}\left[\epsilon_{a}^{\mu} \epsilon_{b}^{\nu}\left(R_{1 \mu \nu}^{a b}+R_{2 \mu \nu}^{a b}\right)+\lambda \phi^{2} \epsilon_{a}^{\mu} U_{\mu}^{a}-4 \phi^{2} \psi^{2} \lambda^{2}\left(P_{1}^{55}+P_{2}^{55}\right)\right] \sqrt{g} d^{4} y .
$$

At this point two possibilities are open. One can either take the action $I$ as a starting point, with all fields independent, and eliminate non-dynamical fields by their equations of motion. Or one can impose the torsion constraint, and derive an action for Levi-Civita connections. We will follow the second approach.

It turns out that, in general, one gets an uninteresting model, describing just two decoupled universes. A more interesting example is obtained by imposing the additional condition $\psi=0$. In other words, we consider on Dirac operators of the form

$$
D=\left(\begin{array}{cc}
\gamma^{a} e_{a}^{\mu} \partial_{\mu}+\cdots & \gamma^{5} \phi(x) \\
\gamma^{5} \phi(x) & \gamma^{a} e_{a}^{\mu} \partial_{\mu}+\cdots
\end{array}\right),
$$

which is in fact closer to the form of Dirac operators used in particle models [1-6].

The zero torsion condition has the following consequences for the components:

1. $\omega_{\mu}^{a b} \equiv \omega_{1 \mu}^{a b}=\omega_{2 \mu}^{a b}$ is the one form corresponding to the classical Levi-Civita connection of the metric $g_{\mu \nu}=e_{\mu}^{a} e_{\nu}^{a}$. It is the unique solution of $d e^{a}+\omega^{a b} \wedge e^{b}=0, \omega^{a b}=-\omega^{b a}$

2. $\ell^{a b}=\ell^{b a}, \ell^{5 a}=-\ell^{a 5}$.

3. $\omega_{1 \mu}^{a 5}=-\omega_{2 \mu}^{a 5}=\lambda^{-1} \ell^{a b} e_{\mu}^{b}$

4. $\partial_{\mu} \lambda=e_{\mu}^{a} \ell^{5 a}$

It is interesting to notice that the zero torsion constraint selects $\mathbb{Z}_{2}$-equivariant connections. In other words, let $\theta: A \rightarrow A$ be the involution $\theta\left(a_{1} \oplus a_{2}\right)=a_{2} \oplus a_{1}$. Extend it, using the equivariance of $D$, to the unique involutive automorphism of $\Omega_{D}(A)$ such that $d \theta=\theta d$. Then Levi-Civita connections have the property $\theta \otimes \theta \nabla=\nabla \theta$. 
The resulting gravity action is then

$$
I=\int_{Y}\left[2 R-\lambda^{-1} 4 \nabla_{\mu} \partial^{\mu} \lambda+4 \lambda^{-2} \ell^{a a} \ell^{55}+\lambda^{-2}\left(\ell^{a a} \ell^{b b}-\ell^{a b} \ell^{a b}\right)\right] \sqrt{g} d^{4} y
$$

The fields $\ell^{a b}, \ell^{55}$ decouple, and with the substitution $\lambda=\exp (\sigma)$, we finally obtain the action of a massless scalar coupled to the gravitational field:

$$
I=2 \int_{Y}\left[R-2 \partial_{\mu} \sigma \partial^{\mu} \sigma\right] \sqrt{g} d^{4} x
$$

To understand the role of the field $\sigma$ we can study the coupling of gravity to the Yang-Mills sector. In particular, in the example of the standard model in [3] we see that

$g^{\mu \nu}$ is the metric of the Riemannian manifold while $\phi=e^{-\sigma}$ replaces the electroweak scale $\mu$. In other words, the vacuum expectation value of the field $\phi$ determines the electroweak scale, thus forming a connection between gravity and the standard model. From the form of the gravity action, it is clear that the field $\sigma$ has no potential. The only other term we could have added is a cosmological constant

$$
\left(E^{A} E^{B}, E^{A} E^{B}\right)
$$

and this is $\sigma$-independent. This implies that at the classical level the vacuum expectation value of $\phi$ is undetermined. It is conceivable that the gravity action acquires a ColemanWeinberg potential through quantum effects. However, at present this is beyond our capabilities, since the problem of quantization in non-commutative geometry has not as yet been dealt with.

\section{Acknowledgements}

We thank $A$. Connes and J. Lott for very helpful discussions and for providing us with an advance copy of [3]. 


\section{References}

[1] A. Connes, in The interface of mathematics and particle physics , Clarendon press, Oxford 1990, Eds D. Quillen, G. Segal and S. Tsou

[2] A. Connes and J. Lott, Nucl. Phys. B Proc. Supp. 18B 29 (1990), North-Holland, Amsterdam.

[3] A. Connes and J. Lott, The metric aspect of noncommutative geometry, to appear in Proceedings of the 1991 Cargèse summer school.

[4] D. Kastler, Marseille preprints

[5] R. Coquereaux, G. Esposito-Farése, G. Vaillant, Nucl. Phys.B353 689 (1991);

M. Dubois-Violette, R. Kerner, J. Madore, J. Math. Phys.31 (1990) 316;

B. Balakrishna, F. Gürsey and K. C. Wali, Phys. Lett. 254B (1991) 430.

[6] A. H. Chamseddine, G. Felder and J. Fröhlich, Grand unification in non-commutative geometry, Zürich, preprint 1992. 